\title{
Workplace Violence against Female Nurses in Menoufia Governorate, Egypt: An Epidemiological Study
}

Hala M Gabr ${ }^{1}$, Faten E Younis ${ }^{1 *}$, Aziza S El-Badry ${ }^{1}$

${ }^{1}$ Public Health and Community Medicine Department, Tanta Faculty of Medicine, Tanta University, Egypt

\begin{abstract}
:
Background: Workplace violence (WPV) against nurses becomes a global problem with negative sequel on healthcare providers. Objectives: to evaluate the prevalence of WPV against Egyptian female nurses and to determine its sociodemographic and occupational risk factors. Methods: This crosssectional study was performed at different level hospitals in Menoufia governorate, Egypt. A total of 1023 nurses were included, with response rate $(94.82 \%)$ : Nine hundred and seventy nurses. They worked on primary $(n=120)$, secondary $(n=380)$ and tertiary $(n=470)$ level of hospitals. Predesigned data collection sheet was prepared to record WPV and its risk factors. Results: The prevalence of WPV was estimated to affect $71.24 \%$ of the studied nurses. They were exposed to verbal, physical, or sexual assault during their professional career $(60.31 \%, 8.66 \%$ and $14.12 \%$; respectively). Also, significant association between the public or tertiary hospital where the participants worked and WPV was found (85.38\% and 52.96\%; respectively). Risk factors for WPV included working in Emergency department, night shift workers, younger nurses, and work experience less than 15 years. Perpetrators of violence were in decreasing order of frequency patients' relatives $(68.88 \%)$, patients $(40.38 \%)$ followed by the head of the work (29.38\%) and finally colleagues (18.09\%). Conclusion: Increased WPV against female nurses become tragic in healthcare settings. Ultimate implementation of protective interventions and behavioral education sessions of nurses is needed.
\end{abstract}

Keywords: Healthcare, Hospitals, Nurses, Violence

Introduction:

Workplace violence (WPV) constitutes a major problem in hospitals with nurses being at a greater risk of abuse. ${ }^{(1,2)}$ About a quarter of the world's WPV occurs in hospitals. ${ }^{(3)}$ The World Health Organization (WHO) defined workplace violence (WPV) as "incidents where working staff are abused, threatened, or assaulted in various circumstances during their work. ${ }^{(4)}$

Most nurses have been exposed to different forms of WPV from patients and/or their relatives, as well as exposure to aggressive behavior from their colleagues, managers and/or patients. WPV included verbal threats, physical and/or sexual assaults by patients and/or their relatives who interact with the medical staff. ${ }^{(5,6)}$ Any of these acts of WPV poses a negative impact on the safety and health of nurses $^{(7)}$ and may disturb the quality of patient healthcare. ${ }^{(8)}$

The aim of this study was to assess the prevalence of WPV against Egyptian female nurses and to determine its sociodemographic and occupational risk factors.

\section{Methods:}

This cross-sectional study was conducted in Menoufia governorate, Egypt from the period of first January to the end December 2019.This study employed multistage random sample. The first stage: Out of 9 districts of Menoufia governorate, two districts (Shebin Elkom and Menouf) were chosen randomly. Second stage involved hospitals selection. From each district, three levels of hospitals comprised of one primary healthcare center, a secondary healthcare hospital and a tertiary healthcare

*Corresponding author: fatenyounis@ yahoo.com

(c) (c) This article is an open access article distributed under the terms and conditions of the Creative Commons Attribution (CC BY) license (http://creativecommons.org/licenses/by/4.0/) 
hospital were chosen randomly. The third stage included all female nurses of any age, working in the selected healthcare facilities and agreed to participate in the study. Total sample size of nurses was 1023, with response rate (94.82\%): Nine hundred and seventy nurses. They worked on primary, secondary, and tertiary care level of hospitals (120, 380 and 470 nurses, respectively).

The tertiary care hospitals had the highest number of participants (470 nurses). This was because tertiary hospital had specialized consultative care, specialized intensive care units and advanced diagnostic support services which provided usually on referral from primary and secondary hospitals. So, it was required higher numbers of nurses than primary or secondary hospitals.

Predesigned data collection sheet was prepared to assess WPV and its risk factors. It was validated by a pilot survey of 10 nurses. This validation aimed to evaluate the time needed to complete the questionnaire and to assure that all the questions are phrased clearly and appropriately for comprehension and to avoid bias that might otherwise affect the results. Moreover, more validation was done for reliability coefficient with Cronbach's Alpha which was of 0.82 . The questions were either of short answered, or multiple check boxes.

The study Variables: Independent variables comprised personal characteristics (age, marital status, and graduation degree) and occupational history (department of work, experience years, rank position and work shift. Dependent variables contained past exposure to different types of violence (physical, verbal, and sexual) Data Management: Data was analyzed using Statistical Package for Social Sciences (SPSS) version 22 (SPSS Inc, Chicago, IL, USA). Descriptive statistics were performed to describe the prevalence of WPV and its types. Chi-square test was used to assess the differences in exposure to violence regarding sociodemographic and occupational characteristics of participants. The association between workplace violence types and perpetrator of violence was tested. Odds ratio (OR) with its $95 \%$ confidence interval (CI) were used for risk estimation. All statistical analyses were two-tailed and $\mathrm{p}$ value $<0.05$ was considered significant.

Ethical Considerations: The study was formally approved by Research Ethics Committee at Menoufia Faculty of Medicine. Also, permission was also sought from hospital managers. The objective of the study was thoroughly explained to hospital managers and all participants and they were assured of confidentiality. Written consent was obtained from all participants.

\section{Results:}

This study was included 970 nurses who were drawn from three levels of hospitals (primary, secondary, and tertiary) located in two districts of Menoufia governorate, Egypt.

The prevalence of WPV was estimated to be $71.24 \%$ of the studied nurses. They were 
exposed to verbal, physical, or sexual assault during their work $(60.31 \%, 8.66 \%$ and $14.12 \%$; respectively). They were exposed to more than one type of WPV as shown in Figure (1a and $1 b)$.

The age of participants less than 25 years ago was significantly exposed to the workplace violence than those with aged ranged from 2540 years. While the age of participants above 40 years, was significantly protected from assaults. More than half of the studied nurses who exposed to WPV had a diploma qualification $(53.83 \%)$ and less than 15 experience years $(58.18 \%)$. As regards to the hospitals' differences, a statistically significant association between the public or tertiary hospital where the participants worked and workplace violence (85.38\% and 52.96\%; respectively). Inside the hospital, nurses working in outpatients and emergency department were more assaulted than other departments as depicted in Table 1.

$66.42 \%$ of sexual assaults were between single nurses while the married nurses were more exposed to the verbal and physical violence $(57.95 \%$ and $53.57 \%$; respectively). The nurses working in emergency found to be significantly associated with being assaulted physically (40.48\%). Correspondingly, experience years less than 15 years among participants and junior nurses were also significantly associated with being exposed to physical, verbal, or sexual workplace violence. Night shift was reported more cases of WPV than day shift. Also, 40.38 and 68.88 percent of assaults were reported to patients and their relatives as perpetrators of WPV while 18.09 and 29.38 percent of assaults were documented to the colleagues and head of the work as revealed in Table 2.

Perpetrators of WPV were in decreasing order of frequency patients' relatives, patients followed by the head of the work and finally colleagues as shown in Figure 2.

\section{Discussion:}

The current study confirmed the high propensity of violence against nurses in Tertiary healthcare hospitals in Menoufia governorate, Egypt.

Workplace violence against nurses is reported in many other countries. ${ }^{(9-15)}$ In this study, female nurses were verbally, physically, or sexually assaulted during their professional career $(60.31 \%, 8.66 \%$ and $14.12 \%$, respectively).

Verbal abuse (83.9\%), verbal threats (27.6\%), physical violence $(21.4 \%)$, sexual abuse (10.8\%), followed by ethnical harassment $(6.1 \%)$ were the commonest types of WPV experienced by the Iranian nurses in a recent cross-sectional study. ${ }^{(16)}$

The prevalence of WPV against nurses was $65.8 \%$; of this, $64.9 \%$ was verbal violence, and physical violence and sexual harassment reported to be $11.8 \%$ and $3.9 \%$, respectively among 15970 Chinese nurses. ${ }^{(17)}$

In the current study, nurses working in Emergency department were found to be 
significantly associated with being assaulted physically in the workplace $(40.48 \%)$.

This is in accordance with large Chinese study which reported that frequent workplace violence occurred primarily in Emergency and Pediatrics departments. ${ }^{(17)}$

Night shift nurses were more prone to suffer from workplace violence (WPV) than day shift in this study which is contrary to that reported by Honarvar et al. ${ }^{16}$ Also, Basfr et al., recently reported higher rate of WPV against morning shift nurses when compared to the evening shift nurses (58.4\% versus $42.3 \%$ respectively). ${ }^{(18)}$ This could be related largely to different population and different studied departments.

Perpetrators of violence in descending order of frequency were patients' relatives (68.88\%), patients $(40.38 \%)$ followed by the head of the work (29.38\%) and finally colleagues (18.09\%) in the current study.

These figures were in accordance with previous studies. According to Honarvar et al, patients' relatives, patients followed by physicians were identified as the sources of WPV in $70.6 \%, 43.1 \%$, and $4.1 \%$ of cases, respectively. ${ }^{(16)}$ Respondents reported that patients' relatives were the main perpetrators in tertiary and county-level hospitals in another study. ${ }^{(17)}$

Violent behavior was mostly exhibited by the patients themselves $(81.3 \%)$ in another recent study conducted in Psychiatric Hospital Settings. ${ }^{18}$ More surprising, nurse managers committed violent behaviors against $54 \%$ of the nurse participants in another Saudi study. ${ }^{(15)}$

Experience years less than 15 years among participants and junior nurses were also significantly associated with being exposed to physical, verbal, or sexual workplace violence in this study.

General nurses, younger than 30 years and with high professional titles as well as those working in shifts are at higher risk of psychological violence as reported by a recent Chinese survey. ${ }^{(19)}$

The type of violence faced was not either statistically associated with professional experience, weekly working hours, or working shift in another recent Brazilian study. ${ }^{(14)}$

Workplace violence significantly negatively affected nurses' job satisfaction and associated with higher nurses' turnover intention in another recent Chinese survey of 1024 nurses. $^{(20)}$

Limitations: This study reflected the prevalence of WPV among the nurses and its risk factors only and did not apply any action plane for protection or reducing WPV. So, future research should focus on protective measures against WPV at individual and institutional levels.

Conclusion: Increased WPV against female nurses become tragic in healthcare settings. Ultimate implementation of protective interventions and behavioral education sessions of nurses is needed.

Acknowledgment: We would like to express our sincere gratitude and deepest appreciation to 
the nurses who accepted to participate in this study.

Fund: The research was conducted with no fund.

Conflict of Interest: There was no conflict of interest.

\section{References:}

1. Nelson R. Tackling violence against health-care workers. The Lancet. 2014. p. 1373-4.

2. Joa TS, Morken T. Violence towards personnel in out-of-hours primary care: A cross-sectional study. Scand J Prim Health Care. 2012;30(1):55-60.

3. Azodo CC, Ezeja EB, Ehikhamenor EE. Occupational violence against dental professionals in Southern Nigeria. Afr Health Sci. 2011;11:1486-92.

4. Wang S, Hayes L, O'Brien-Pallas L. A review and evaluation of workplace violence prevention programs in the health sector. Univ Toronto [Internet]. 2008; Available from: http://www.mtpinnacle.com/pdfs/RevWork-violence-MOHLTC-2008.pdf

5. Di Martino V. Workplace violence in the health sector Country case studies Thailand and an additional Australian study by. 2002;49. Available from: https://www.who.int/violence_injury_pr evention/violence/activities/workplace/ WVsynthesisreport.pdf?ua=1
6. Kaya S, Demir IB, Karsavuran S, Ürek D, Ilgün G. Violence against doctors and nurses in Hospitals in Turkey. J Forensic Nurs. 2016;12(1):26-34.

7. Magnavita N. The exploding spark: Workplace violence in an infectious disease hospital - A longitudinal study. Biomed Res Int. 2013;1-9.

8. Henneman EA, Roche JP, Fisher DL, Cunningham H, Reilly CA, Nathanson $\mathrm{BH}$, et al. Error identification and recovery by student nurses using human patient simulation: Opportunity to improve patient safety. Appl Nurs Res. 2010;23(1):11-21.

9. Jiao M, Ning N, Li Y, Gao L, Cui Y, Sun $\mathrm{H}$, et al. Workplace violence against nurses in Chinese hospitals: A crosssectional survey. BMJ Open. 2015;5(3):e006719.

10. Sisawo EJ, Ouédraogo SYYA, Huang SL. Workplace violence against nurses in the Gambia: mixed methods design. BMC Health Serv Res. 2017;17(1):311.

11. Hassankhani H, Parizad N, Gacki-Smith J, Rahmani A, Mohammadi E. The consequences of violence against nurses working in the emergency department: A qualitative study. Int Emerg Nurs. 2018;39:20-5.

12. Ramacciati N, Ceccagnoli A, Addey B, 
Rasero L. Violence towards Emergency Nurses. The Italian National Survey 2016: A qualitative study. Int J Nurs Stud. 2018;81:21-9.

13. Chang YP, Lee DC, Wang HH. Violenceprevention climate in the turnover intention of nurses experiencing workplace violence and work frustration. J Nurs Manag. 2018;26(8):961-71.

14. Cavalcanti AL, Belo E dos R, Marcolino E de C, Fernandes A, Cavalcanti YW, de CARVALHO DF, et al. Occupational violence against brazilian nurses. Iran J Public Health. 2018;47(11):1636-43.

15. Rayan A, Sisan M, Baker O. Stress, Workplace Violence, and Burnout in Nurses Working in King Abdullah Medical City During Al-Hajj Season. J Nurs Res. 2019;27(3):e26.

16. Honarvar B, Ghazanfari N, Shahraki HR, Rostami S, Lankarani KB. Violence against nurses: A neglected and healththreatening epidemic in the university affiliated public hospitals in Shiraz, Iran. Int J Occup Environ Med. 2019;10(3):111-23.
17. Shi L, Zhang D, Zhou C, Yang L, Sun T, Hao T, et al. A cross-sectional study on the prevalence and associated risk factors for workplace violence against Chinese nurses. BMJ Open. 2017;7(6):e013105.

18. Basfr W, Hamdan A, Al-Habib S. Workplace violence against nurses in psychiatric hospital settings perspectives from Saudi Arabia. Sultan Qaboos Univ Med J. 2019;19(1):e19-25.

19. Li P, Xing K, Qiao H, Fang H, Ma H, Jiao $\mathrm{M}$, et al. Psychological violence against general practitioners and nurses in Chinese township hospitals: Incidence and implications. Health Qual Life Outcomes. 2018;16(1):117.

20. Zhao SH, Shi Y, Sun ZN, Xie FZ, Wang JH, Zhang SE, et al. Impact of workplace violence against nurses' thriving at work, job satisfaction and turnover intention: A cross-sectional study. J Clin Nurs. 2018;27(13-14):2620-32. 
Table (1): Association between Sociodemographic and Occupational Characteristics of nurses with Violence Prevalence

\begin{tabular}{|c|c|c|c|c|c|}
\hline $\begin{array}{c}\text { Sociodemographic } \\
\text { and occupational } \\
\text { characteristics }\end{array}$ & $\begin{array}{c}\text { Violence } \\
(\text { No.=691) }\end{array}$ & $\begin{array}{l}\text { No violence } \\
\text { (No.=279) }\end{array}$ & $\chi^{2}$ test & P value & $\begin{array}{c}\text { Odds ratio } \\
(95 \% \text { CI })\end{array}$ \\
\hline $\begin{aligned} \text { Age } & \\
. & <25 \text { year } \\
\text {. } & 25-40 \text { year } \\
\text {. } & >40 \text { year }\end{aligned}$ & $\begin{array}{l}324(46.89 \%) \\
198(28.65 \%) \\
169(24.46 \%) \\
\end{array}$ & $\begin{array}{l}82(29.39 \%) \\
94(33.69 \%) \\
103(36.92 \%)\end{array}$ & 76.93 & $<0.001$ & $\begin{array}{c}1.88(1.33-2.65) \\
\text { Reference } \\
0.78(0.55-1.10)\end{array}$ \\
\hline $\begin{array}{cl}\text { Marital status } \\
\text { - Single } \\
\text { - Married } \\
\text { - } \text { Widow or } \\
\text { divorced } \\
\end{array}$ & $\begin{array}{c}261(37.78 \%) \\
382(55.28 \%) \\
48(6.94 \%)\end{array}$ & $\begin{array}{c}98(35.12 \%) \\
155(55.56 \%) \\
26(9.32 \%)\end{array}$ & 1.85 & 0.397 & ------- \\
\hline $\begin{array}{c}\text { Graduation degree } \\
\text { - Diploma } \\
\text { - Bachelor } \\
\text { degree }\end{array}$ & $\begin{array}{l}372(53.83 \%) \\
319(46.17 \%)\end{array}$ & $\begin{array}{c}80(28.67 \%) \\
199(71.33 \%)\end{array}$ & 50.56 & $<0.001$ & $\begin{array}{c}2.90(2.15-3.91) \\
\text { Reference }\end{array}$ \\
\hline $\begin{array}{ll}\text { Department } \\
\text { - Outpatients } \\
\text { - Emergency } \\
\text { - Medical } \\
\text { - Surgical } \\
\text { - ICU } \\
\end{array}$ & $\begin{array}{c}137(19.83 \%) \\
163(23.59 \%) \\
146(21.13) \\
159(23.01 \%) \\
86(12.44 \%) \\
\end{array}$ & $\begin{array}{c}35(12.54 \%) \\
53(19.00) \\
52(18.64 \%) \\
71(25.45 \%) \\
68(24.37 \%) \\
\end{array}$ & 26.74 & $<0.001$ & $\begin{array}{c}1.39(0.86-2.27) \\
1.10(0.70-1.71) \\
0.80(0.52-1.22) \\
0.45(0.29-0.71) \\
\text { Reference }\end{array}$ \\
\hline $\begin{array}{c}\text { Experience years } \\
\text { - } \quad<15 \text { years } \\
\text { - }>15 \text { years } \\
\end{array}$ & $\begin{array}{l}402(58.18 \%) \\
289(41.82 \%) \\
\end{array}$ & $\begin{array}{c}83(29.75 \%) \\
196(70.25 \%)\end{array}$ & 64.25 & $<0.001$ & $\begin{array}{c}3.28(2.44-4.42) \\
\text { Reference }\end{array}$ \\
\hline $\begin{array}{c}\text { Rank position } \\
\text { - } \text { Senior } \\
\text { - } \text { Junior }\end{array}$ & $\begin{array}{l}272(39.36 \%) \\
419(60.64 \%)\end{array}$ & $\begin{array}{l}133(47.67 \%) \\
146(52.33 \%)\end{array}$ & 5.64 & 0.017 & $\begin{array}{c}\text { reference } \\
1.40(1.06-1.86)\end{array}$ \\
\hline \begin{aligned} & \multicolumn{2}{l}{ Hospital } \\
& - Public \\
& - Private \\
&\end{aligned} & $\begin{array}{l}590(85.38 \%) \\
101(14.62 \%)\end{array}$ & $\begin{array}{c}198(70.97 \%) \\
81(29.03 \%)\end{array}$ & 27.10 & $<0.001$ & $\begin{array}{c}2.39(1.71-3.34) \\
\text { Reference }\end{array}$ \\
\hline $\begin{array}{c}\text { Work shift } \\
\text { - Day } \\
\text { - Night } \\
\end{array}$ & $\begin{array}{l}295(42.69 \%) \\
396(57.31 \%)\end{array}$ & $\begin{array}{l}167(59.86 \%) \\
112(40.14 \%)\end{array}$ & 23.48 & $<0.001$ & $\begin{array}{c}\text { Reference } \\
2.00(1.51-2.66)\end{array}$ \\
\hline $\begin{array}{c}\text { Type of hospital } \\
\text { - Primary } \\
\text { - Secondary } \\
\text { - Tertiary }\end{array}$ & $\begin{array}{c}63(9.12 \%) \\
262(37.92 \%) \\
366(52.96 \%) \\
\end{array}$ & $\begin{array}{c}57(20.43 \%) \\
118(42.29 \%) \\
104(37.28 \%) \\
\end{array}$ & 31.63 & $<0.001$ & $\begin{array}{c}0.50(0.33-0.76) \\
\text { Reference } \\
1.58(1.17-2.16) \\
\end{array}$ \\
\hline
\end{tabular}


Table (2): Association between Sociodemographic and Occupational Characteristics of Nurses with Types of Violence

\begin{tabular}{|c|c|c|c|c|c|c|}
\hline \multirow{2}{*}{$\begin{array}{l}\text { Sociodemographic } \\
\text { and occupational } \\
\text { characteristics }\end{array}$} & \multirow[t]{2}{*}{ Total } & \multicolumn{3}{|c|}{ Violence } & \multirow[t]{2}{*}{$\chi^{2}$ test } & \multirow[t]{2}{*}{ P value } \\
\hline & & $\begin{array}{c}\text { Verbal } \\
(\text { No.=585) }\end{array}$ & $\begin{array}{l}\text { Physical } \\
\text { (No.= 84) }\end{array}$ & $\begin{array}{c}\text { Sexual } \\
(\text { No. }=137)\end{array}$ & & \\
\hline $\begin{aligned} \text { Age } & \\
\text {. } & <25 \text { year } \\
\text {. } & 25-40 \text { year } \\
\text {. } & >40 \text { year }\end{aligned}$ & $\begin{array}{l}324 \\
198 \\
169\end{array}$ & $\begin{array}{l}284(48.54 \%) \\
176(30.09 \%) \\
125(21.37 \%) \\
\end{array}$ & $\begin{array}{l}36(42.86 \%) \\
26(30.95 \%) \\
22(26.19 \%)\end{array}$ & $\begin{array}{l}63(45.98 \%) \\
51(37.23 \%) \\
23(16.79 \%) \\
\end{array}$ & 4.65 & 0.325 \\
\hline \begin{tabular}{cl}
\multicolumn{2}{l}{ Marital status } \\
- & Single \\
- & Married \\
- & Widow or \\
& divorced \\
\end{tabular} & $\begin{array}{c}261 \\
382 \\
48\end{array}$ & $\begin{array}{c}207(35.38 \%) \\
339(57.95 \%) \\
39(6.67 \%)\end{array}$ & $\begin{array}{l}24(28.57 \%) \\
45(53.57 \%) \\
15(17.86 \%)\end{array}$ & $\begin{array}{c}91(66.42 \%) \\
41(29.93 \%) \\
5(3.65 \%)\end{array}$ & 61.95 & $<0.001$ \\
\hline $\begin{array}{cl}\text { Graduation degree } \\
\text { - Diploma } \\
\text { - } \text { Bachelor } \\
\text { degree }\end{array}$ & $\begin{array}{l}372 \\
319\end{array}$ & $\begin{array}{l}301(51.45 \%) \\
284(48.55 \%)\end{array}$ & $\begin{array}{l}51(60.71 \%) \\
33(39.29 \%)\end{array}$ & $\begin{array}{l}74(54.01 \%) \\
63(45.99 \%)\end{array}$ & 2.26 & 0.27 \\
\hline $\begin{array}{l}\text { Department } \\
\text { - Outpatients } \\
\text { - Emergency } \\
\text { - Medical } \\
\text { - Surgical } \\
\text { - ICU } \\
\end{array}$ & $\begin{array}{l}137 \\
163 \\
146 \\
159 \\
86 \\
\end{array}$ & $\begin{array}{c}97(16.58 \%) \\
157(26.84 \%) \\
105(17.95 \%) \\
151(25.81 \%) \\
75(12.82 \%) \\
\end{array}$ & $\begin{array}{c}14(16.67 \%) \\
34(40.48 \%) \\
12(14.28 \%) \\
17(20.24 \%) \\
7(8.33 \%) \\
\end{array}$ & $\begin{array}{c}30(21.90 \%) \\
33(24.09 \%) \\
37(27.01 \%) \\
26(18.97 \%) \\
11(8.03 \%) \\
\end{array}$ & 19.24 & 0.013 \\
\hline $\begin{array}{c}\text { Experience years } \\
\text { - } \quad<15 \text { years } \\
\cdot \quad>15 \text { years } \\
\end{array}$ & $\begin{array}{l}402 \\
289 \\
\end{array}$ & $\begin{array}{l}342(58.46 \%) \\
243(41.54 \%) \\
\end{array}$ & $\begin{array}{l}53(63.09 \%) \\
31(36.91 \%) \\
\end{array}$ & $\begin{array}{c}116(84.67 \%) \\
21(15.33 \%) \\
\end{array}$ & 32.87 & $<0.001$ \\
\hline $\begin{array}{c}\text { Rank position } \\
\text { - Senior } \\
\text { - } \text { Junior } \\
\end{array}$ & $\begin{array}{l}272 \\
419\end{array}$ & $\begin{array}{l}182(31.11 \%) \\
403(68.89 \%)\end{array}$ & $\begin{array}{l}20(23.81 \%) \\
64(76.19 \%)\end{array}$ & $\begin{array}{l}76(55.47 \%) \\
61(44.53 \%)\end{array}$ & 33.90 & $<0.001$ \\
\hline \begin{aligned} \multicolumn{2}{l}{ Hospital } \\
- Public \\
- Private \\
\end{aligned} & $\begin{array}{l}590 \\
101\end{array}$ & $\begin{array}{c}546(93.33 \%) \\
39(6.67 \%)\end{array}$ & $\begin{array}{c}82(97.62 \%) \\
2(2.38 \%)\end{array}$ & $\begin{array}{l}49(35.77 \%) \\
88(64.23 \%)\end{array}$ & 286.59 & $<0.001$ \\
\hline $\begin{array}{c}\text { Work shift } \\
\text { - Day } \\
\text { - Night } \\
\end{array}$ & $\begin{array}{l}295 \\
396\end{array}$ & $\begin{array}{l}246(45.13 \%) \\
339(54.87 \%)\end{array}$ & $\begin{array}{l}24(28.57 \%) \\
60(71.43 \%)\end{array}$ & $\begin{array}{l}51(37.23 \%) \\
86(62.77 \%\end{array}$ & 6.03 & 0.049 \\
\hline $\begin{array}{c}\text { Type of hospital } \\
\text { - Primary } \\
\text { - Secondary } \\
\text { - Tertiary }\end{array}$ & $\begin{array}{c}63 \\
262 \\
366\end{array}$ & $\begin{array}{c}42(7.18 \%) \\
259(44.27 \%) \\
284(48.55 \%)\end{array}$ & $\begin{array}{c}2(2.38 \%) \\
27(32.14 \%) \\
55(65.48 \%)\end{array}$ & $\begin{array}{l}23(16.78 \%) \\
51(37.23 \%) \\
63(45.99 \%)\end{array}$ & 24.44 & $<0.001$ \\
\hline
\end{tabular}




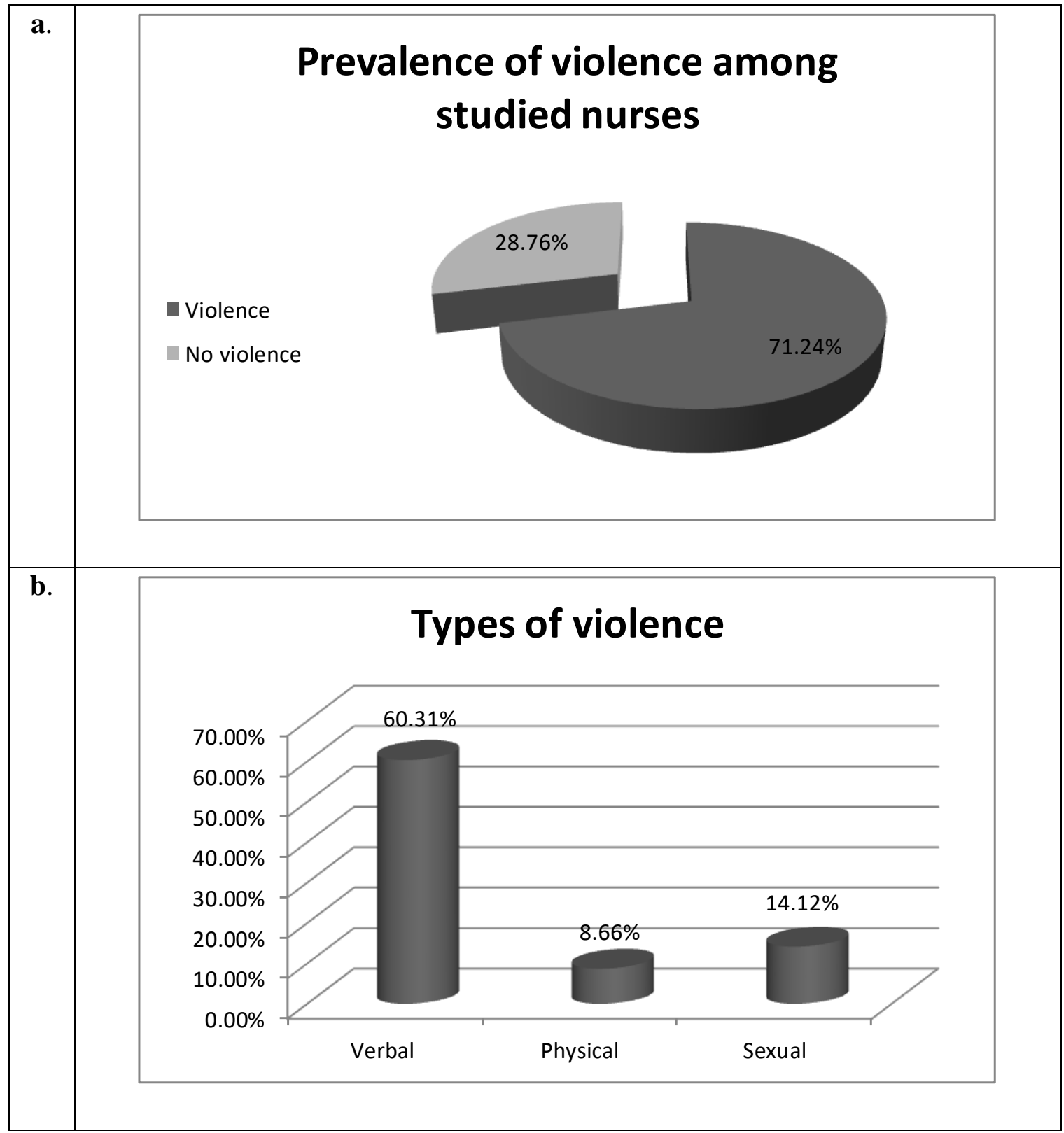

Figure (1): The Prevalence of Workplace Violence and its Types among Nurses 


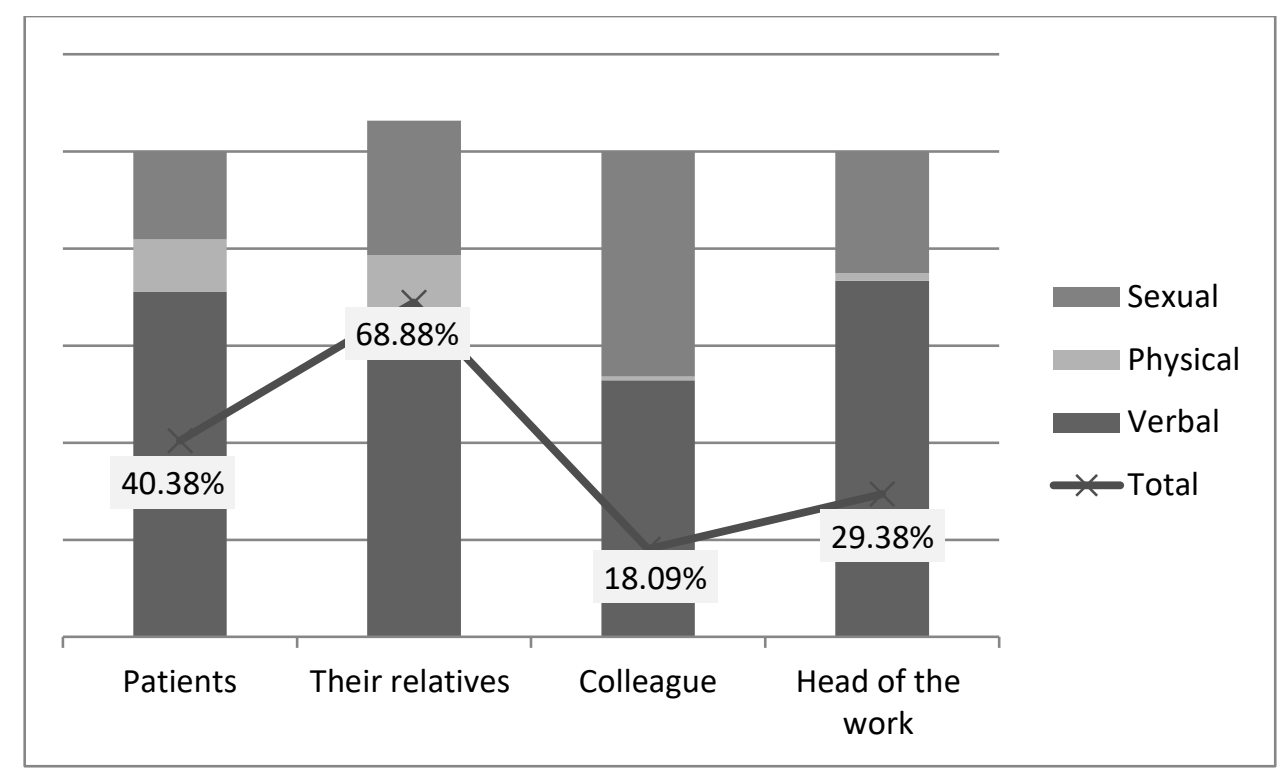

Figure (2): The Perpetrators of Workplace Violence among Nurses 


\section{العنف في مكان العمل ضد الممرضات في محافظة المنوفية بمصر: دراسة وبائية}

هاله مروان جبر ـ فاتن عزالعرب يونس - عزيزة سعد البدري

المقدمة: أصبحت ظاهرة العنف في مكان العمل ضد الممرضات منتشرة مع توابعها السلبية على مقدمى الخدمة الطبية. الهدف: لتحديد معدل أنتشار ظاهرة العنف في مكان العمل ضد المررضات المصريات وتحديد عو امل الخطورة لهذه الظاهرة

طرق البحث: تم اجر اء هذه الدر اسة المستعرضة على المستويات المختلفة لمستشفيات الخدمة الصحية فى محافظة المنوفية بمعدل أستجابة بلغ 94.82\% . شملت الدر اسة970 ممرضة من العاملات بمستشفيات الرعاية الصحية الأولية والثانوية و المستوى الثالث

حيث تم ملئ أستبيان سابق التصميم للبحث عن معدل أنتشار و أسباب العنف في مكان العمل ضد الممرضات. النتائج: بلغ معدل أنتشار ظاهرة العنف في مكان العمل ضد الممرضات 71.24\% بين الممرضات المشاركات فى الأستبيان وتباين بين أعتداء لفظى وجسدى وتعدى جنسى وتم الأعتداء غالبا فى مستشفيات المستوى الثالث من الرعاية الصحية. كانت أبرز عو امل الخطورة لأنتشار هذه الظاهرة: العمل فى قسم الطوارئ، العمل بالمناوبات الليلية، الأصغر سنا من الممرضات و الأقل خبرة من 15 عام بين الممرضات. وكان مرتكبو العنف مرتبين تنازليا كالأتى: أقارب المرضى، المرضى أنفسهم، رؤساء العمل وأخير ا الزملاء فى العمل. الخلاصة والتوصيات: أصبح أنتشار العنف في مكان العمل ضد الممرضات أمر ا مأساويا مما يستدعى سر عة التدخل لايجاد أليات حماية وبر امج تتقيف سلوكى مكثفة. 